\title{
A Study on Developing Image Processing for Smart Traffic Supporting System Based on AR
}

\author{
Byeong-Wook Nam, Kyung-Ho Lee, Jung-Min Lee \\ Dept. Naval Architecture and Ocean Engineering, Inha University \\ Nam-Gu Inha-Ro 100, Incheon, Korea \\ nbw0519@naver.com; kyungho@inha.ac.kr;jm80.lee@gmail.com
}

\begin{abstract}
This paper suggests a smart traffic supporting system on the vessel based on augmented reality technology. International Hydrographic Organization(IHO) adopted standard S-100 as the international standard Geographic Information System(GIS) that can be generally used in the maritime sector. The smart traffic supporting system means system that obtains the information of the real world through a camera and visualizes traffic data on the image based on S-100. At this time, there is a need for a variety of image processing techniques in order to obtain a reliable information from the image obtained through the camera. In this study, we performed the image processing that can process an image noise which is generated by the operating environment and can detect an object in order to support safe traffic. finally, we verified the technology by developing a prototype.
\end{abstract}

Keywords: Smart traffic supporting system, Augmented reality, Image processing, e-Navigation

\section{Introduction}

Since the $20^{\text {th }}$ century, as the worldwide maritime the quantity of goods transported increases[1], shipbuilding techniques which is larger and speed of the ship were developed to support this. Accordingly, the sail technology capable of controlling the large vessels were also developed.

For the maritime sector data processing, International Hydrographic Organization(IHO) established international standard S-52 for data representation and international standard S-57 for Electric Navigation Chart(ENC) production. The Electric Navigation Chart(ENC) digitalizing paper navigation chart and Electric Chart Display and Information System(ECDIS) were introduced as initial navigation support equipment and systems based on that.

Recently, the International Maritime Organization(IMO) has introduced the next generation of electronic navigation system for the purpose of maritime safety and marine environment protection. there was a limit in the conventional data processing standard S-57 to integrate and utilize various aspects of maritime and land. Accordingly, IMO developed S-100 that is marine data processing standard for overcoming this. The S-10x which are the details standards of S-100 are expected to be converted by the new electronic charts. According to this trend, in this study, we developed smart traffic supporting system based on augmented reality(AR) for supporting navigation using S-10x that is becoming the standard.

If the vessel is operated close to the coast or there are obstructions around the vessel, an officer determines by comparing visually the navigational equipment and marine conditions. At this time, even as for a short time, officer is placed in the state cannot confirm the maritime situation depending on the line of sight of officer facing down. When an officer in an unstable visibility, according to the officer fails to confirm the maritime situation with the naked eye, the officer is to rely on radar and ECDIS equipment, resulting in a higher probability of accidents.

In this study, we tried to develop a system that can support the traffic based on the augmented reality technology. As augmented reality technology is a technology that can be rolled up for sophisticated visualization of the virtual information based on a real image, the technology allows the officer to help determine the maritime situation and traffic information at the same time. In this paper, we deal about the image processing for obtaining traffic information based on the image processing prior to development the smart traffic supporting system, constructed a prototype based on this and verify its validity. 


\section{Related Works}

Sub-committee on safety of navigation(NAV) 54th Work group report annex I of the IMO shows the state of the environment when e-Navigation is complete. Also it mentions the importance with this. In addition, a study of the eNavigation is made to effectively display the navigation information, and The US Navy has introduced a system called Augmented Reality Visualization of the Common Operational Picture(ARVCOP)[2].

\subsection{ARVCOP}

ARVCOP was developed by TSI Corporation. The US Navy was to be visible on the screen by integrating the various navigational equipment for the successful support of military operations in any situation. In particular, this system was to be able to perform a successful mission by providing information for routes, operations performed utilizing augmented reality technology in the weather unstable visibility. it was estimated that there is possible to improve the operation and the concentration at the time of forward-looking through the system. There is continuous research and development that has been made by increasing the importance of operation information.

\subsection{Image-based Object Detection and Tracking Techniques on the Marine Environment}

It has been made many studies for the detection of objects in a marine environment. System which can identify the ship using ARPA radar and CCTV image have been developed by Lee[3]. Park et al. have developed a system for keeping tracking of the detected object by using the Kalman filter[4]. In the marine environment, unlike road environment, the recognition of a distant target is essential. When tracking a target by using the image processing, if the target is more than a certain distance away from the camera, it is impossible to detect the target. Therefore, it is necessary to apply a filter considering the direction of the movement in order to compensate for this. This filter may provide the location to track a target through a prediction without having the detection for a space. In this study, we have to detect and identify objects in the marine environment. Because it can be more accurate tracking if a known target is detected, we additionally used the information in the ship automatic identification system (AIS).

\section{AR based Smart Traffic Supporting System}

Visualized traffic information consists of the own-ship information, target-ship information, route information, aids to navigation information, environmental information, and steering characteristic information. The traffic-related information may be provided integrated with ECDIS. In addition, the position and the speed of the ship is obtained through a global positioning system(GPS). Maritime mobile service identification(MMSI) number and sailing status is acquired through the vessel AIS device. The image of the sea conditions is input by using a camera and then this image was processed to identify the target. This processed image is matched with the traffic information is provided to the user.

\subsection{Overview}

AR technology is a visualization technique that more than one information at the same time on one screen, elaborately matching information of the virtual computer-generated based on the real world can be provided to the user. AR has been the research in the aerospace, automotive industries and so on, as an intermediate form of virtual reality and the real world. Recently, these fusion research is being conducted in the shipbuilding. For AR based show traffic information, it should be carried out based on the camera image. After receiving the input image officer is looking through the camera, and recognizes the target through the image processing. By effectively visualizing the information obtained from the navigation equipment on the image, it is possible to reduce the time the user's gaze is fixed down. By projecting the virtual information on the screen, it can provide a sense of security to officer in an unstable situation of visibility. Figure 1 shows the concept of AR based smart traffic supporting system to be presented in this paper. 


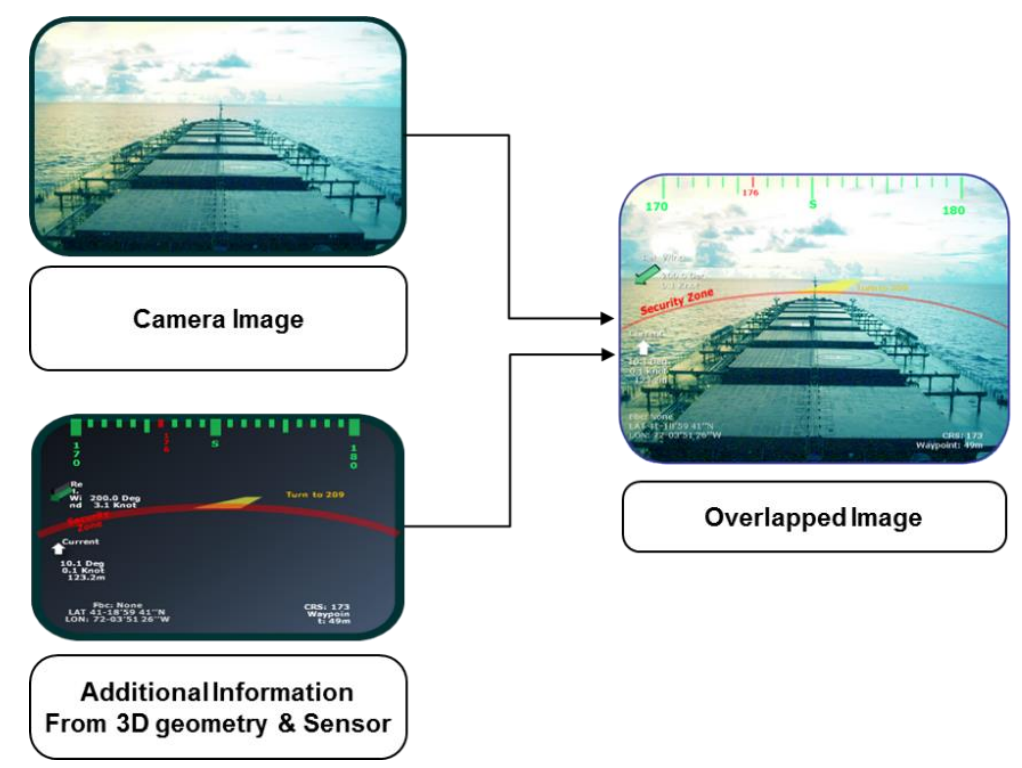

Fig. 1: Concept of smart traffic supporting system.

\subsection{Configuration}

Figure 2 shows the information for configuring the AR based smart traffic supporting system to be proposed in this paper. Traffic images can be obtained through a CCTV camera. This study mainly targets that the vessel has the bow bridge, in other words, the vessel to and from the coast. the vessel has the stern bridge, in other words, since vessel, such as container ship has a component which can affect the accuracy of the image processing to the CCTV images, there is a need for additional image processing techniques. The image obtained through the CCTV camera includes a lot of noise characteristics the marine environment. This image is subjected to image pre-processing for detecting a horizontal and objects. Image pre-processing includes processes such as Gaussian blur and image smoothing. After image processing such as segmentation, the information such as horizontal line and objects can be detected. In this paper, this process is called image processing. It is possible to visualize with the information of the object by mapping the detected result and the AIS information. Most of the navigation information, including various sensors for traffic attached to the vessel, can be obtained through ECDIS. However, we utilize only the decoded AIS data in order to verify its validity in this study.

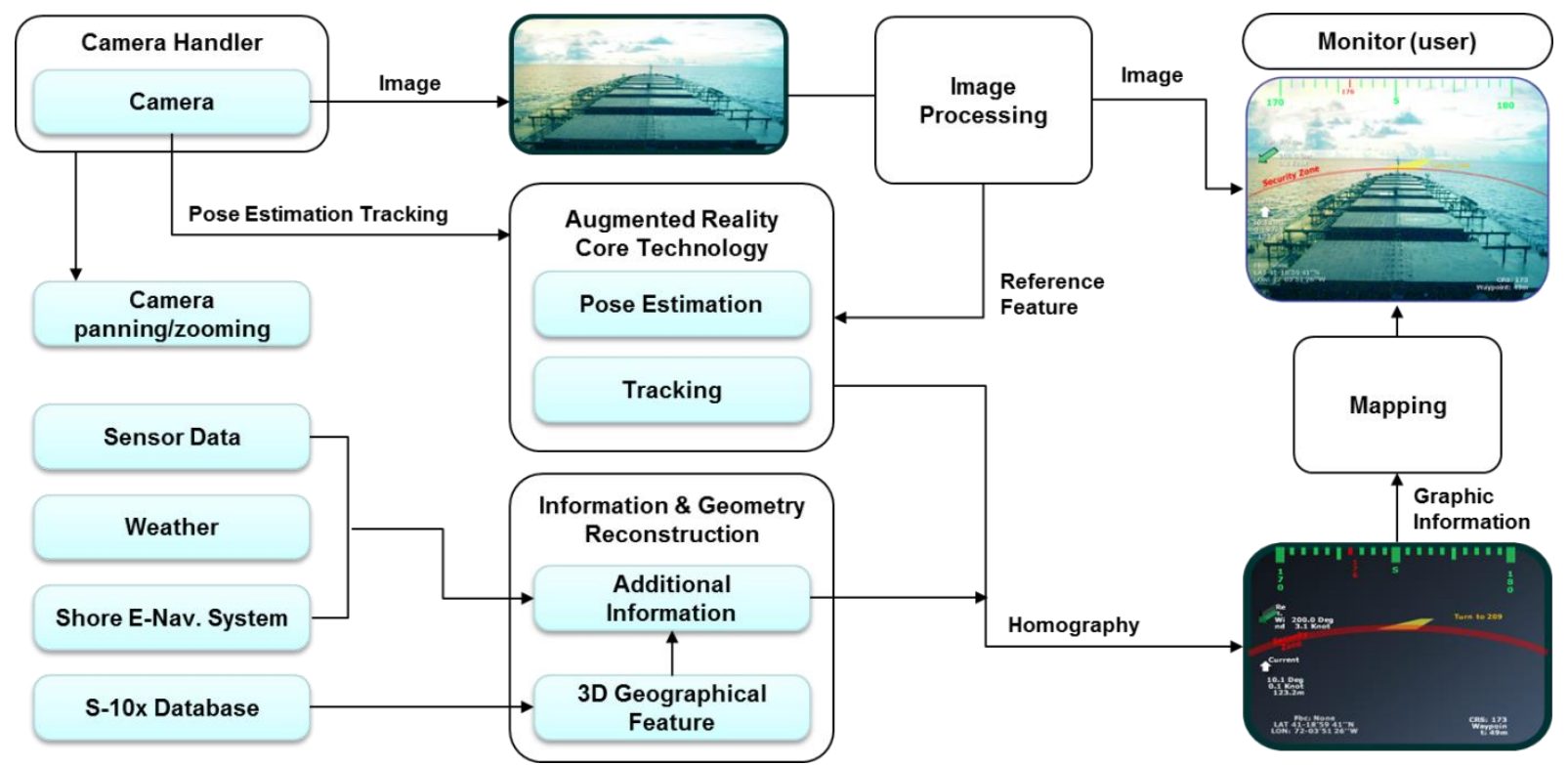

Fig. 2: Configuration of smart traffic supporting system. 


\section{Image Analysis for Detecting the Traffic Information}

This study was divided into three steps for image analysis step, such as horizontal line detection, object detection and object recognition. In this paper, we analysed the image by image processing in order to visualize the traffic information. This results are compared to the traffic information, which was screened to the target. Image analysis includes an image pre-processing step. The main output of the module is the position information of the object through image analysis and horizontal line position. Figure 3 is showing a detailed flow of the module.

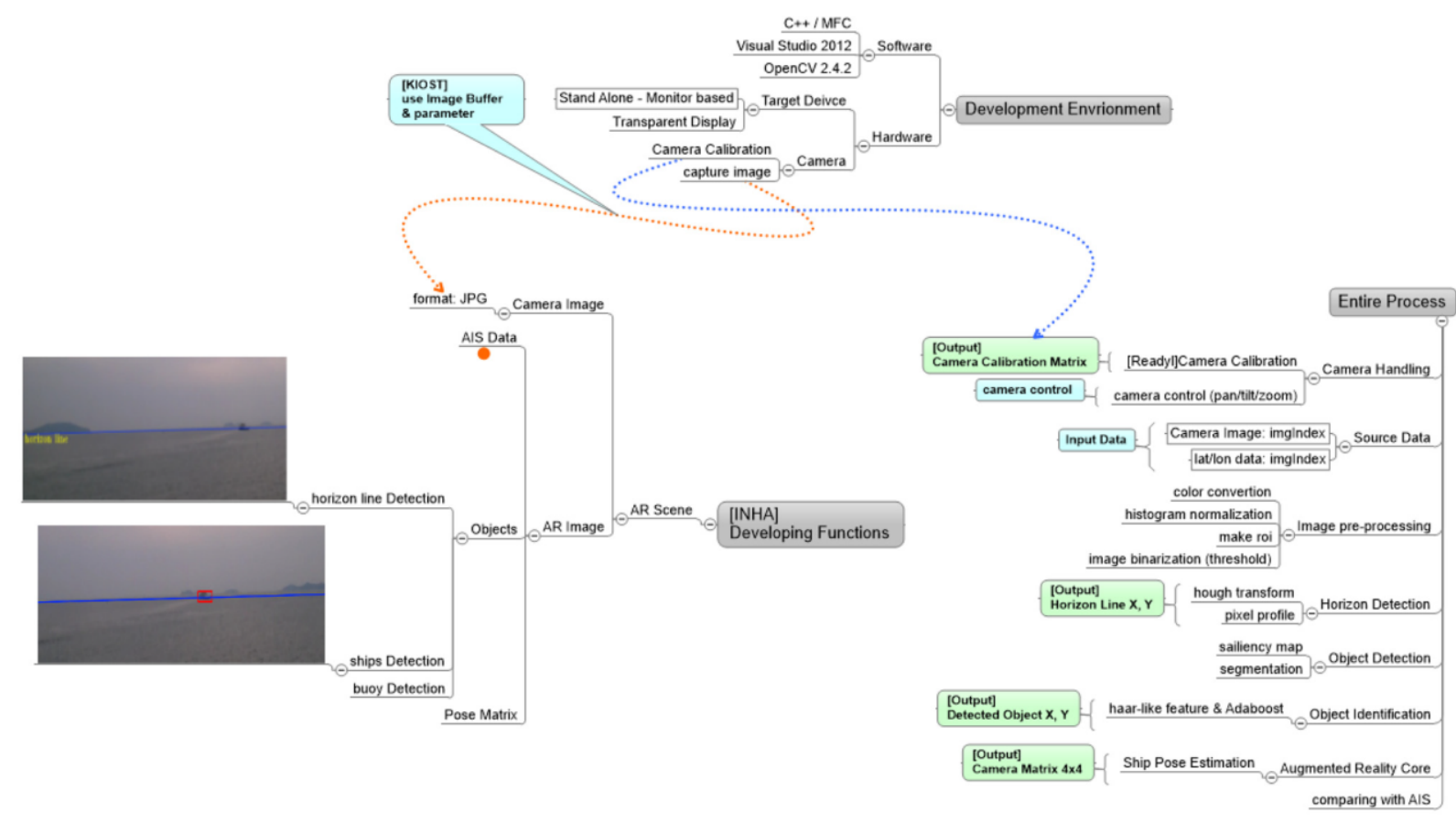

Fig. 3: Development configuration of the image processing module.

\subsection{Detection of Horizontal Line}

Because it is not possible to image processing in order to obtain the location information of the ship is currently underway, The AIS, Gyrocompass, including GPS information is required. In additional, if information is visualized utilizing this information, the error that officers are looking at the screen will occur. Therefore, mapping the various traffic information through the image processing and object recognition process, it will be able to deliver more accurate information to the officer. In this paper, it was to set the Region of Interest(ROI) of the image by detecting the horizontal line in order to detect a horizontal line in the effective image. ROI for detecting the horizontal line may be applied differently according to the position of the camera installed in the vessel. Figure 4 is showing a key step in the process for detecting the horizontal line. Horizontal line is generally present in the shade of rapidly changing point. Therefore,

(a) Switch the camera images in black and white image in order to perform image processing.

(b) Smooth the histogram in order to clearly image.

(c) And then it performs binarization using the threshold value calculated automatically.

(d) It is possible to detect the horizontal line applying Canny edge and Hough transform. 


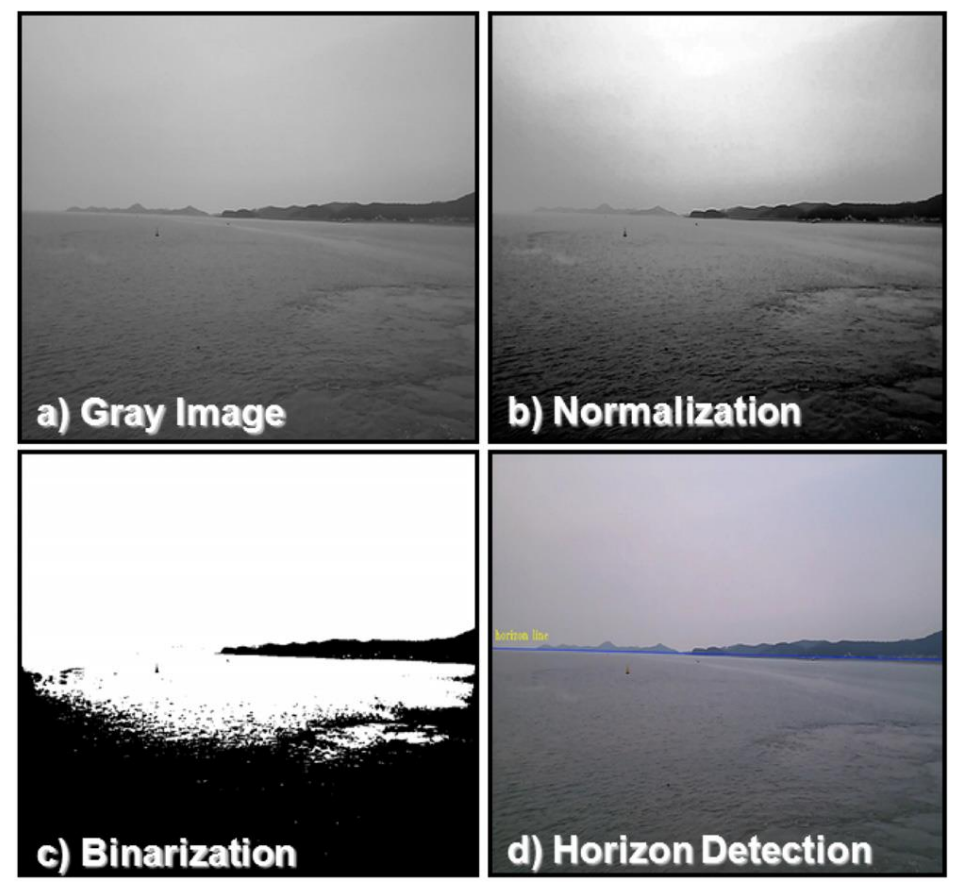

Fig. 4: Horizontal line detection process.

This can apply the ROI to enhance the detection accuracy of the horizontal line, and it is possible to perform in Hue Saturation Value(HSV) space. Figure 5 is a result of detection by applying the ROI in a horizontal line is unclear situation. The weather having blurred vision, such as fog is difficult to recognize on the horizon. According to the image processing method, in particular, it is brought back to a variety of errors according to the value of the critical point. The horizontal line is basically in accordance with the draft of the ship is always fixed, or change the width of a small, storing the initial position of the horizon to the ROI area can be set. In addition, since the detection of the horizontal line find the width of the brightness variation where the highest priority, if the brightness difference, for reasons such as fog, is placed below the horizontal line, it will fail to detect. Thus, if the ROI area is set in order to correct this, it is possible to detect a more accurate horizontal line.

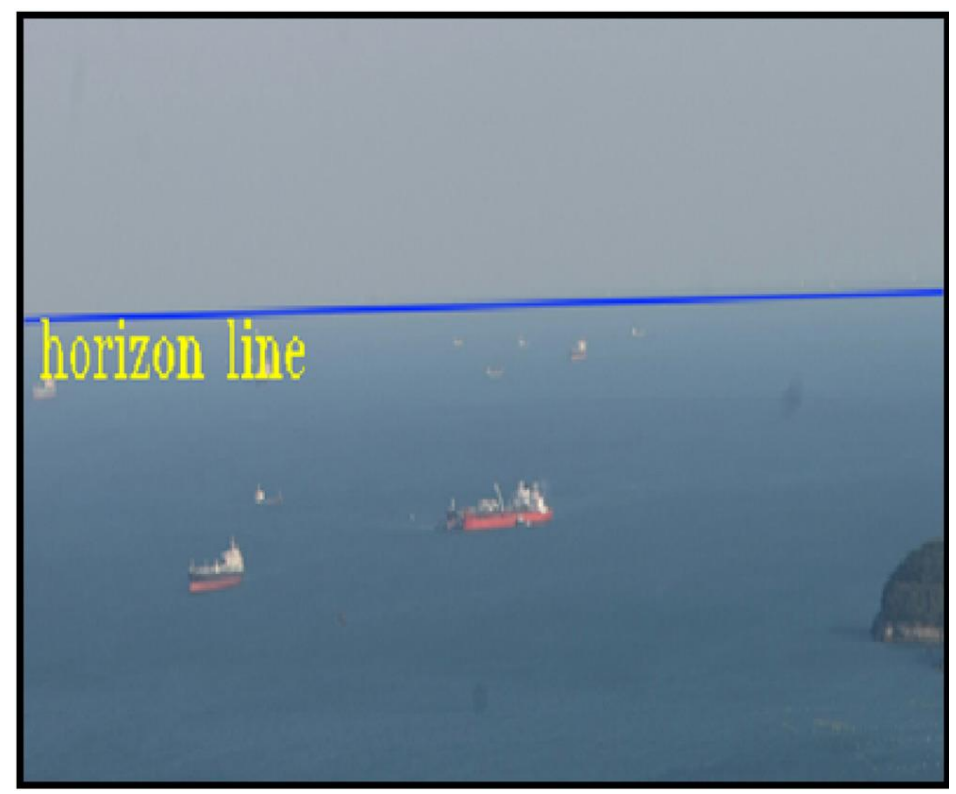

Fig. 5: Horizontal line detection by using ROI. 


\subsection{Detection of Objects}

The detected horizontal line can be used to filter objects. Because the object is under the majority of the maritime horizontal line, object existing in the position above the horizontal line can be ignored or cannot be detected. Reflective effect of light at sea, wave, and trajectory generates noise. This is a factor that makes it difficult for the image processing. In the figure 6, image can be seen that a lot of noise at the bottom. This is likely to be detected as the object that are not required to detect the object. It used the method of Gaussian blurring for removing noise for this. However, using the blur on an object far away in the marine environment, a problem that the object is gone with the noise is generated. Therefore, in order to detect more efficiently the object, the object was to detect by utilizing the map Saliency[5]. Saliency map model is based on the finding that the gross area of the differences of the colour, brightness, and contours in the image as a topdown way to fine an area of interest from the first image processing. Figure 6 is showing the object detection process, (a) is CCTV image, (b) is the converted saliency map image, (c) shows a binarization processing result, (d) is labeling perform result. According to the results of figure 6, the centre of the image seems far vessel and the right side of the image shows the nearest island. Because the top of the image has a relatively snarled difference in brightness due to a sunburst, this has been reflected in saliency map. this can be removed by performing filtering after detecting the horizontal line.

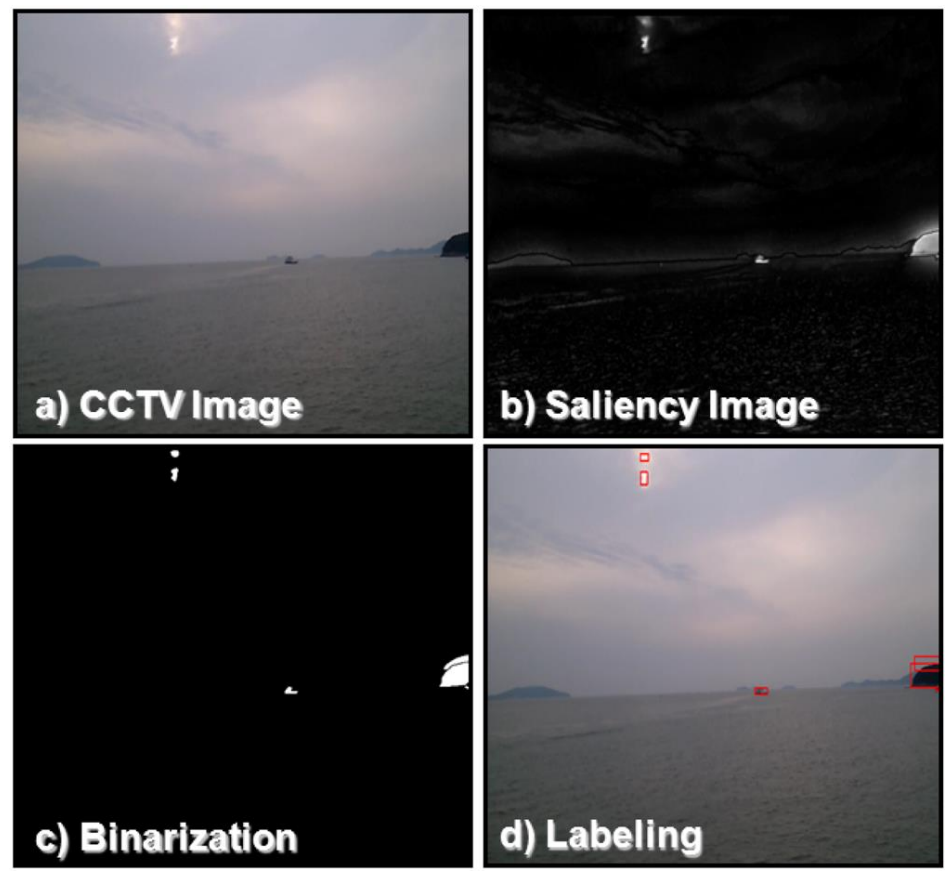

Fig. 6: Object detection process.

\subsection{Objects Recognition}

In order to map the information about the detected target, MMIS, latitude and longitude values of the AIS was utilized. Focusing the camera installed on the vessel, objects in Field of View(FOV) of CCTV camera are sorted. AIS is basically using GPS information. If using the same satellite, there is also known that the same error occurring. Differential GPS(DGPS) is also using the GPS information. Therefore, a distance is calculated by using the latitude and longitude can be used in the filtering using the FOV. In this paper, there is a concern only for the object in view of the current user in order to visualize the traffic information. So we carried out the image processing to detect the object. Since image processing results contains only the information about the specific area visible in the image, these areas have to determine whether there is a need for an object. Accordingly, we hope to filter it by comparing the AIS information about the current user viewing area. figure 7 shows the object filtering using the camera FOV. After listing the objects in the centre of the current ship, filtering only information in the area of interest. 

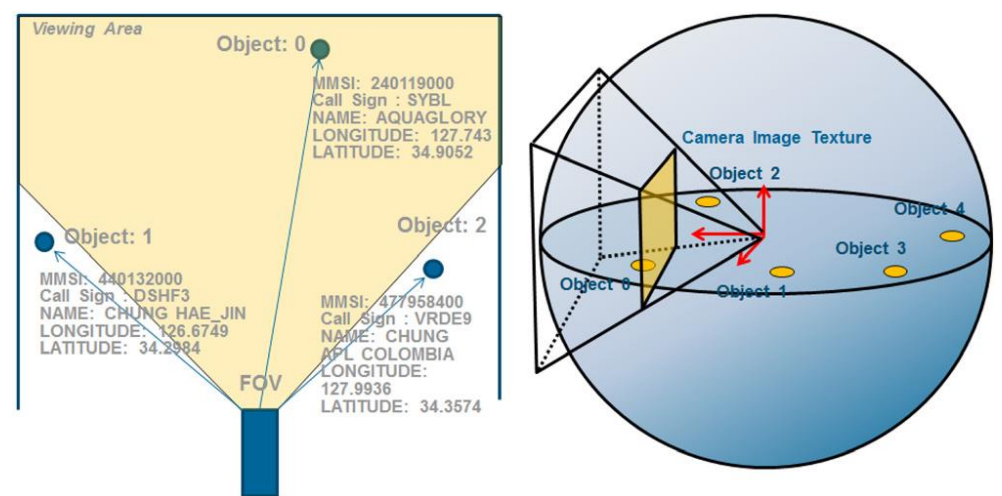

Fig. 7: Object filtering by using FOV.

\section{Results of Image Analysis}

Current ROI was defined as $35 \%$ in the middle of the image. The figure 8 shows the results of the object of marine buoy recognition result of the horizontal line detection. This image was utilized image taken from vessel to and from Yeongjong-Do and Sin-Do, in Korea. The higher accuracy of an object utilizing Saliency map model than the accuracy of the acquired object in the binarization process using the threshold. We could easily filter the detected object in the image there is only one object such as Figure 8. But if a plurality of objects is detected, the accuracy was dropped such as Figure 6. In particular, in order to distinguish each ships, buoys, and birds such as seagull, it seems filter that can distinguish the target is to be applied in addition with respect to the labelling image. To compensate for this, we will apply a recognition algorithm using Adaboost and Harrlike feature[6].

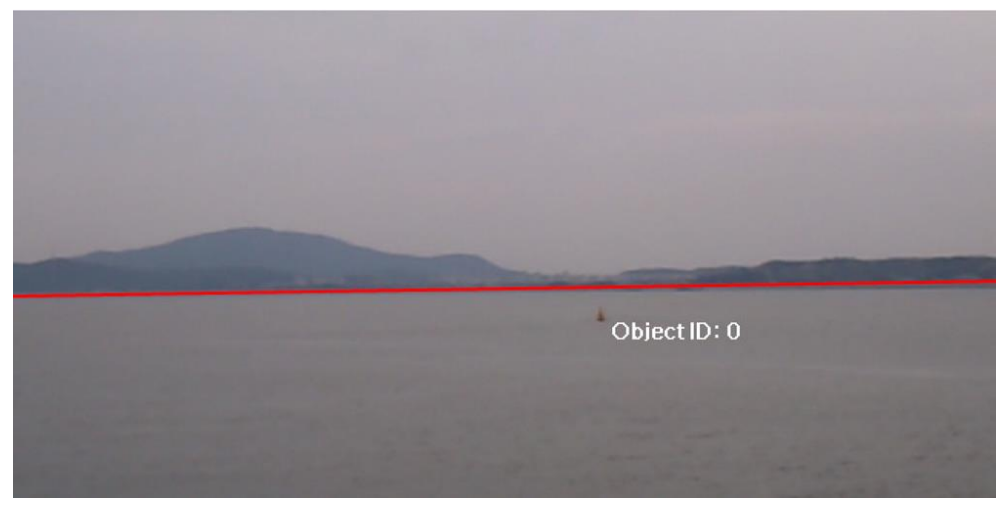

Fig. 8: Horizon and object identification.

\section{Conclusion}

This study is the intermediate result of a study utilizing the augmented reality technology that allows the officers can see the marine environment and the traffic information at the same time, we extracted the information from the traffic image based on the image processing, studied image analysis for visualizing traffic information based on AR technology. Module detecting an object above the horizon and sea surface is realized, and object recognition module utilizing AIS information is realized. In the future, we will complement the object recognition algorithm in order to improve the detection accuracy of the object using the Adaboost. And it will express the relationship between the camera coordinate system and the real world coordinates to homograph to represent the 3D traffic information. We will study the interface for obtaining various information from the ECDIS. Before applying to the transparent display, it will be applied to build a CCTV based prototype. 


\section{Acknowledgements}

This material is based upon work supported by the Ministry of Trade, Industry \& Energy (MOTIE, Korea) under Industrial Technology Innovation Program. No. 10067423, Development of an optimized operating system for transporters.

\section{References}

[1] H. S. Kim. Shipping Statics Handbook, Korea Maritime Institute.

[2] C. Benton, "The Tactical Application of Integrated C4ISR and Navigation Systems," Technology Systems Inc.

[3] D. J. Lee, "Automatic Identification of ARPA Radar Tracking Vessels by CCTV Camera System," in Journal of the Korea Society of Fisheries Technology, vol. 45, no. 3, pp. 177-187, 2009.

[4] J. H. Park, J. H. Kim., N. S. Son, "A Study on Vision-based Object Detection and Tracking in a Marine Environment," in Proceeding of KAOSTS, Daegu, Korea, pp. 510-513, 2012.

[5] L. Itti, C. Koch, E. Niebur, "A Model of Saliency-Based Visual Attention for Rapid Scene Analysis," in IEEE Transactions on Pattern Analysis and Machine Intelligence, vol. 20, no. 11, pp. 1254-1259, 1998.

[6] P. Viola, M. J, "Rapid Object Detection Using a Boosted Cascade of Simple Features," Mitsuvishi Electric Research Laboratories, TR2004-043, [Online]. Available: http://www.merl.com/ 